Musées, Patrimoine et Culture scientifiques et techniques

Les expositions temporaires au musée de l'Homme - Le musée comme lieu de débat sur les questions de société

\title{
Anne Stephan
}

\section{OpenEdition}

\section{Journals}

\section{Édition électronique}

URL : http://journals.openedition.org/ocim/1616

DOI : 10.4000/ocim.1616

ISSN : 2108-646X

\section{Éditeur}

OCIM

\section{Édition imprimée}

Date de publication : 1 janvier 2016

Pagination : 8-10

ISSN : 0994-1908

\section{Référence électronique}

Anne Stephan, «Les expositions temporaires au musée de l'Homme - Le musée comme lieu de débat sur les questions de société », La Lettre de I'OCIM [En ligne], 163 | 2016, mis en ligne le 01 janvier 2017, consulté le 02 mai 2019. URL : http://journals.openedition.org/ocim/1616 ; DOI : 10.4000/ocim.1616

Ce document a été généré automatiquement le 2 mai 2019.

Tous droits réservés 


\title{
Les expositions temporaires au musée de l'Homme - Le musée comme lieu de débat sur les questions de société
}

\author{
Anne Stephan
}

\section{Des expositions thématiques sur l'Homme et les sociétés, ses origines et son devenir}

1 La programmation des quatre prochaines années illustre la diversité des champs possibles en résonance avec les thèmes abordés dans la Galerie de l'Homme et, de façon plus engagée, en lien avec des préoccupations sociétales :

- Chroniques d'une renaissance,

17 octobre 2015-13 juin 2016;

- Des préjugés au racisme : l'exclusion de l'autre

(titre de travail), 31 mars 2017-8 janvier 2018 ;

- Néandertal entre mythe et réalité : l'autre et nous

(titre de travail), avril-décembre 2018 ;

- Alimentation (titre de travail), avril-décembre 2019.

La présence des chercheurs et des collections sur le site, la synergie entre les disciplines à l'œuvre au sein du Centre de recherche sur l'évolution de l'Homme et des sociétés, constituent des atouts majeurs pour initier et nourrir les thématiques des expositions temporaires sur des sujets transversaux. Allant des périodes préhistoriques à nos jours, croisant systématiquement biologie et sciences humaines et conjuguant les apports des sciences de l'Homme, de la Société, de la Vie et de la Terre, la pertinence des thèmes 
proposés devrait constituer une offre attractive pour intéresser de nouveaux publics, une raison de venir ou de revenir au musée.

Par la diversité des disciplines convoquées, par l'actualité et la transversalité des sujets, les expositions temporaires sont envisagées en coproduction, notamment avec des musées et des organismes de recherches. Elles ont également vocation à sortir des murs pour être présentées dans des musées de civilisations, de sciences et de société, sur le plan national ou européen, voire international.

\section{Chroniques d'une renaissance, l'exposition d'ouverture}

L'expo-installation Chroniques d'une renaissance (17 octobre 2015-13 juin 2016) anticipe les questions des nouveaux visiteurs comme celles des nostalgiques de l'ancien musée. Elle dévoile les coulisses du chantier qui s'est déroulé à l'abri des murs du musée. Le commissariat de l'exposition a été confié à deux scientifiques du Muséum national d'Histoire naturelle: Serge Bahuchet, ethnobiologiste, directeur du département "Hommes, Natures et Sociétés" et du laboratoire "Éco-Anthropologie et Ethnobiologie" et François Sémah, préhistorien, directeur de l'Enseignement, de la Pédagogie et des Formations, tous deux commissaires de la dernière exposition temporaire La saga de l'Homme présentée avant la fermeture du musée de l'Homme en 2009. Le parcours, réalisé par l'atelier d'architecture Projectiles à la manière d'une installation in situ, se décline en trois séquences.

\section{La fabrique d'un musée : "Que s'est-il passé depuis la fermeture du musée de l'Homme en 2009 ?"}

12 Le site, classé monument historique, devait être conservé à l'identique, tout en s'adaptant aux besoins du nouveau musée. Si de l'extérieur, rien ne semble avoir changé, l'aménagement du pavillon de tête et des différents niveaux de l'aile courbe a été conçu comme une "couche intelligente" et modulable ajoutée à l'existant. Le bâtiment, qui résulte de l'imbrication de deux édifices construits pour les expositions universelles de 1878 et 1937, a accueilli successivement deux musées: le musée d'Ethnographie du Trocadéro en 1882 et le musée de l'Homme en 1938.

13 À l'occasion de la réouverture du musée de l'Homme en 2015, pour rendre compte de la fabrique d'un musée du XXI ${ }^{e}$ siècle, les architectes Olivier Brochet et Emmanuel Nebout et la scénographe Zette Cazalas ont été invités à dérouler le processus de rénovation architecturale du bâtiment et de conception scénographique de la Galerie de l'Homme. À la manière d'une table d'architecte, chacun des récits de chantier se déploie dans sa temporalité, avec sa grammaire spécifique : modularité et réversibilité pour le bâtiment, mise en scène d'un questionnement anthropologique et des collections pour le parcours permanent. En miroir, à la manière d'un paysage, une fresque vidéo reprend ces thèmes dans un travelling introduisant les acteurs de cette rénovation. 


\section{Les archives de l'Humanité : "Quelles collections pour le nouveau musée de l'Homme ?"}

Pour raconter l'Homme et son évolution, le nouveau musée de l'Homme, site faisant partie intégrante du Muséum national d'Histoire naturelle, se devait de rendre visible les collections nationales de référence qu'il conserve. Avec plus de 736000 objets et ensembles, ces collections contribuent à la connaissance de l'évolution humaine sur les dimensions biologiques, culturelles et sociales. La recherche scientifique fait ainsi appel aux collections qui, en retour, s'enrichissent grâce à la diversité des terrains de recherche. Une frise chronologique met en perspective l'histoire du musée de l'Homme, classé Musée de France. Elle met l'accent sur l'héritage scientifique, institutionnel et culturel, depuis le Jardin du Roi, créé en 1635, jusqu'à nos jours. C'est l'occasion de se remémorer les figures du musée de l'Homme que sont Paul Rivet et Georges-Henri Rivière, ainsi que les grands événements et expositions qui en ont marqué l'histoire du musée de l'Homme. Une sélection d'objets illustre les dates clés de cette chronologie, entre artefacts et évocations muséographiques, collections fondatrices et dons.

Pour illustrer le soutien de la Société des Amis du musée de l'Homme (SAMH) à l'enrichissement des collections, il a été choisi de présenter un tambour de pluie provenant de Chine ou du Nord Vietnam de la collection Maratier. En 2009, Claire Maratier, adhérente et fidèle amie de la SAMH, a fait don de 51 objets récoltés par son mari Amédée Maratier. Parmi ces pièces, 38 ont rejoint les collections d'ethnologie du musée de l'Homme - à l'image de ce tambour de pluie - et 15 sont entrées dans les collections de préhistoire. Depuis sa création en 1914, la SAMH a fait rentrer au musée de l'Homme plus de 800 objets de préhistoire et d'ethnologie en provenance des pays les plus divers.

Plus loin, une sélection de "100 objets pour représenter l'Homme" permet au visiteur de mieux appréhender ces archives de l'Humanité que sont les collections d'anthropologie culturelle et de préhistoire conservées au musée de l'Homme et de comprendre la chaîne de traitement, de la collecte à la conservation. Les objets présentés dans des mobiliers recréant l'univers des réserves sont regroupés selon trois thématiques: l'Homme dans son enveloppe corporelle - L'Homme dans son milieu naturel - L'Homme dans la société. Pour clore cette section, les visiteurs sont invités à découvrir un aspect peu connu des collections: une table ronde est dédiée au patrimoine immatériel avec des enregistrements réalisés par des ethnomusicologues et des ethnolinguistes sur le terrain de recherche des scientifiques du musée de l'Homme.

\section{L'ABC de l'Homme : "Quels seront les thèmes abordés par le nouveau musée de l'Homme ?"}

17 Cette séquence, manifeste du musée de l'Homme, se présente sous la forme d'un abécédaire de $\mathrm{A}$ comme Afrique et Alimentation mais aussi Adaptation à $\mathrm{Z}$ comme Zéro. Pour évoquer cet exemple d'un musée appréhendant l'Homme dans ses dimensions biologiques, sociales et culturelles ainsi que dans ses relations avec la nature, l'abécédaire offre une forme ouverte, multiple et subjective pour aborder les thèmes de recherche de ce musée-laboratoire. Ces thèmes ont présidés à la conception de la Galerie de l'Homme et 
contribueront à la programmation des futures expositions temporaires. Ils recouvrent les grands enjeux liés à l'évolution humaine: histoire et évolution de l'espèce humaine, impacts et interactions de l'Homme avec son milieu, définition et complexité de l'humain, diversité des représentations de l'Homme et de la nature, diversité des cultures matérielles et des sociétés humaines... Les 26 lettres de l'alphabet, associées à un ou plusieurs mots clés - et qui pourraient en illustrer bien d'autres - sont autant d'occasions de rencontres avec les chercheurs et les membres du comité d'orientation du musée de l'Homme, spécialistes reconnus dans leur domaine. Les séquences, extraites du filmalphabet $A B C$ de l'Homme (auteur et réalisateur: Andres Jarach. 26 séquences de 2 à 3 minutes, une production Les Films d'Ici 2, Muséum national d'Histoire naturelle, Bachibouzouk, avec la participation de France Télévisions 2015), prennent place dans une forêt de lettres géantes, de $\mathrm{A}$ à $\mathrm{Z}$, déployées dans un espace ludique.

\section{Un artiste invité, Pascale Marthine Tayou, grand assembleur}

Dans le cadre de l'exposition d'ouverture, le musée de l'Homme accueille l'artiste Pascale Marthine Tayou, né en 1966 au Cameroun, représenté par Galleria Continua. Au début de sa carrière, Pascale Marthine Tayou prend un double nom au féminin : Pascal (e) Marthin (e). Il prend ainsi une distance ironique avec l'idée d'artiste démiurge, la catégorisation homme/femme et toute limitation géographique ou culturelle. Ses installations, étonnants assemblages, entre nt en résonance avec les thématiques abordées dans le parcours permanent sur la globalisation et l'hybridation des cultures. Elles jalonnent un parcours dans les espaces de l'exposition temporaire et dans les différents lieux d'accueil du musée. À cette occasion, Jean-Hubert Martin, directeur honoraire du musée d'Art moderne Centre Pompidou et commissaire de nombreuses expositions, selon lequel "l'idée communément admise qu'il n'y a de création en arts plastiques que dans le monde occidental ou fortement occidentalisé est à mettre au compte des survivances de l'arrogance de notre culture", a été sollicité par le musée de l'Homme pour proposer les lignes de force de la présence d'artistes dans les espaces d'expositions du musée. 
Récits de chantier du processus de rénovation du musée de l'Homme et ses acteurs

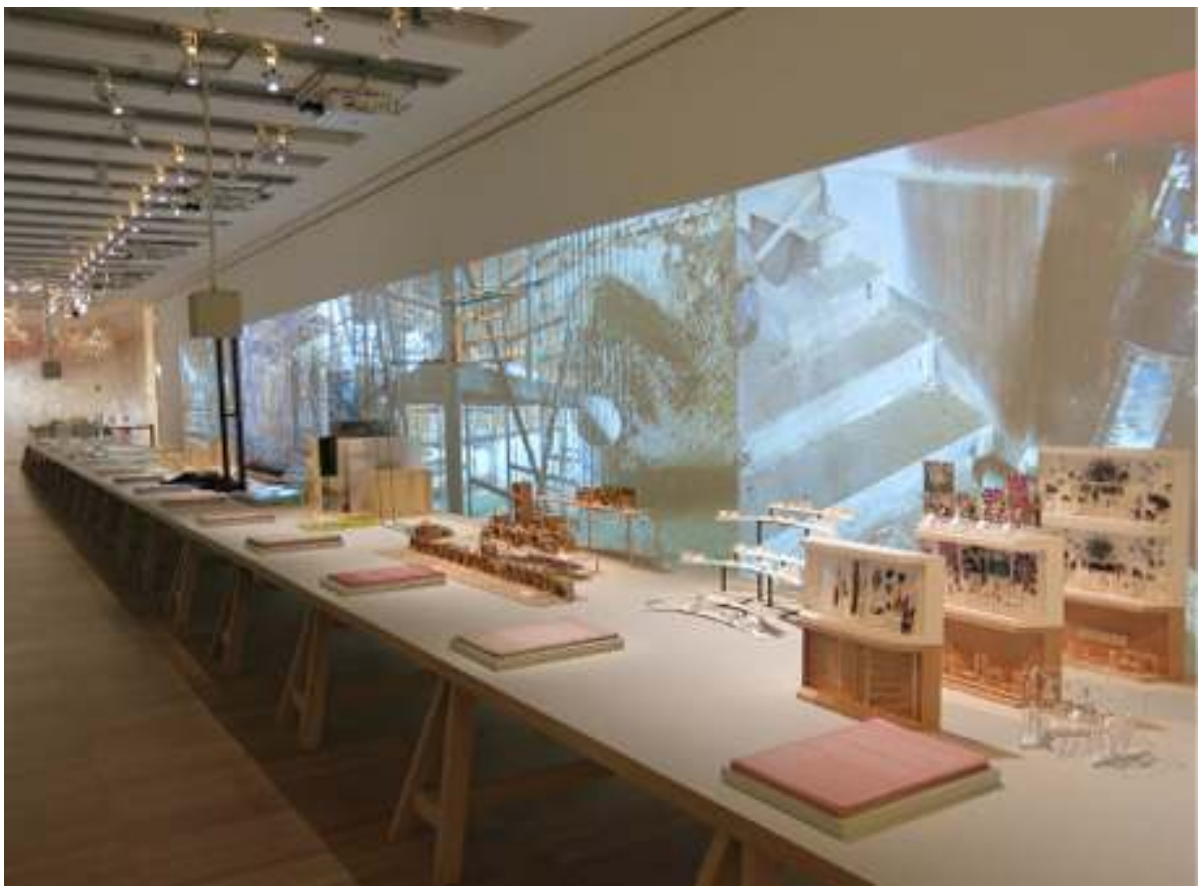

(C) MNHN/J-C Domenech

L'exposition Chroniques d'une renaissance anticipe les questions des nouveaux visiteurs comme celles des nostalgiques de l'ancien musée.

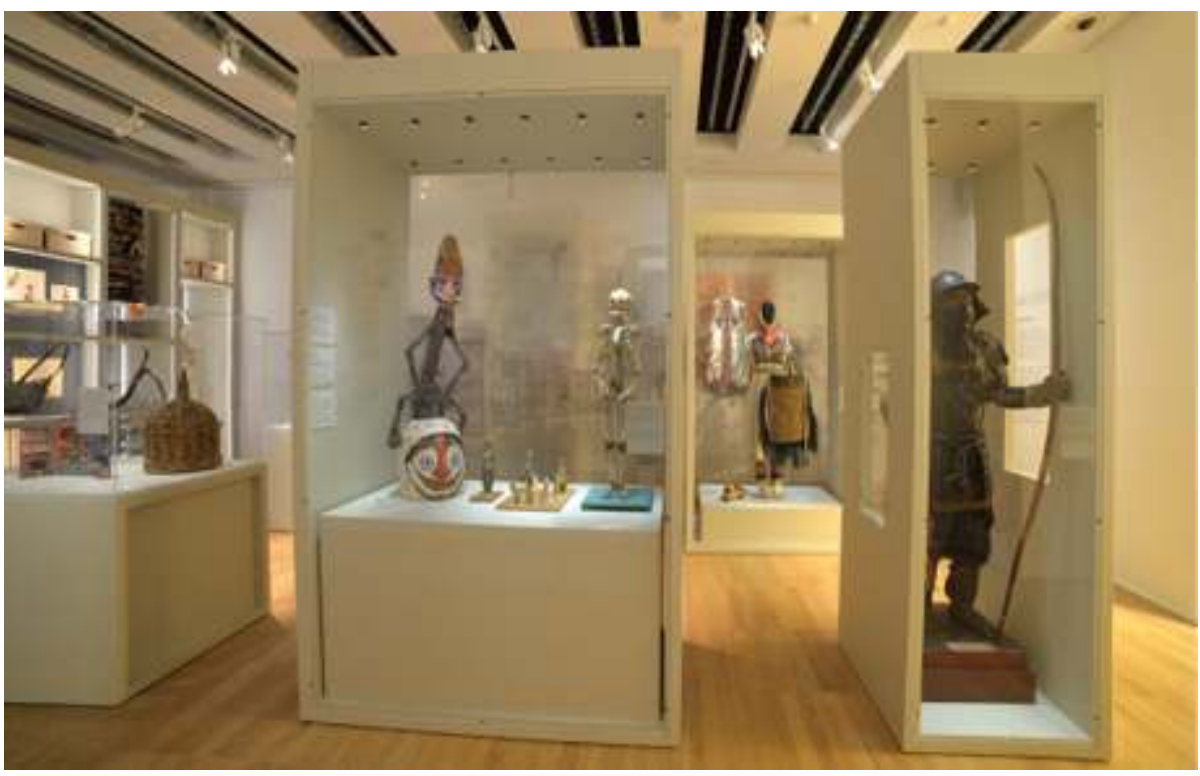

(c) MNHN/J-C Domenech 
Forêt de lettres géantes qui illustre, de A à Z, les thèmes abordés par le musée de l'Homme.

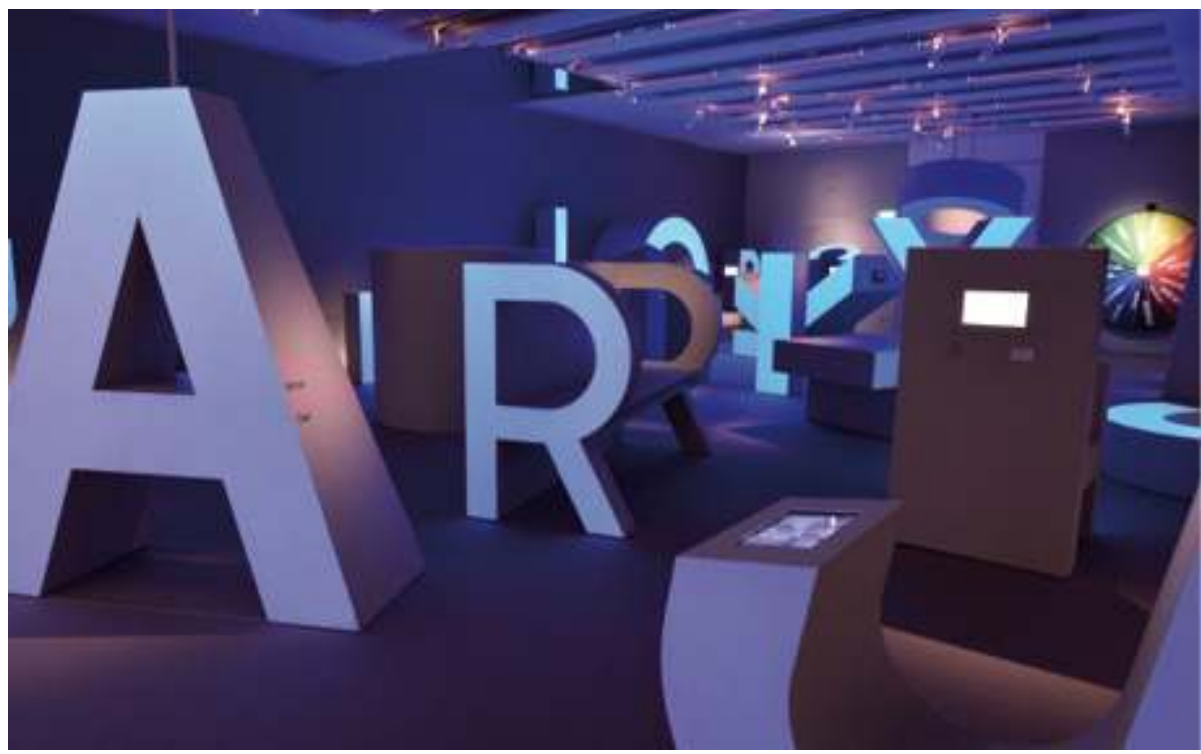

(C) MNHN/J-C Domenech

Un artiste invité, Pascale Marthine Tayou, grand assembleur, dont les installations jalonnent les espaces du musée.

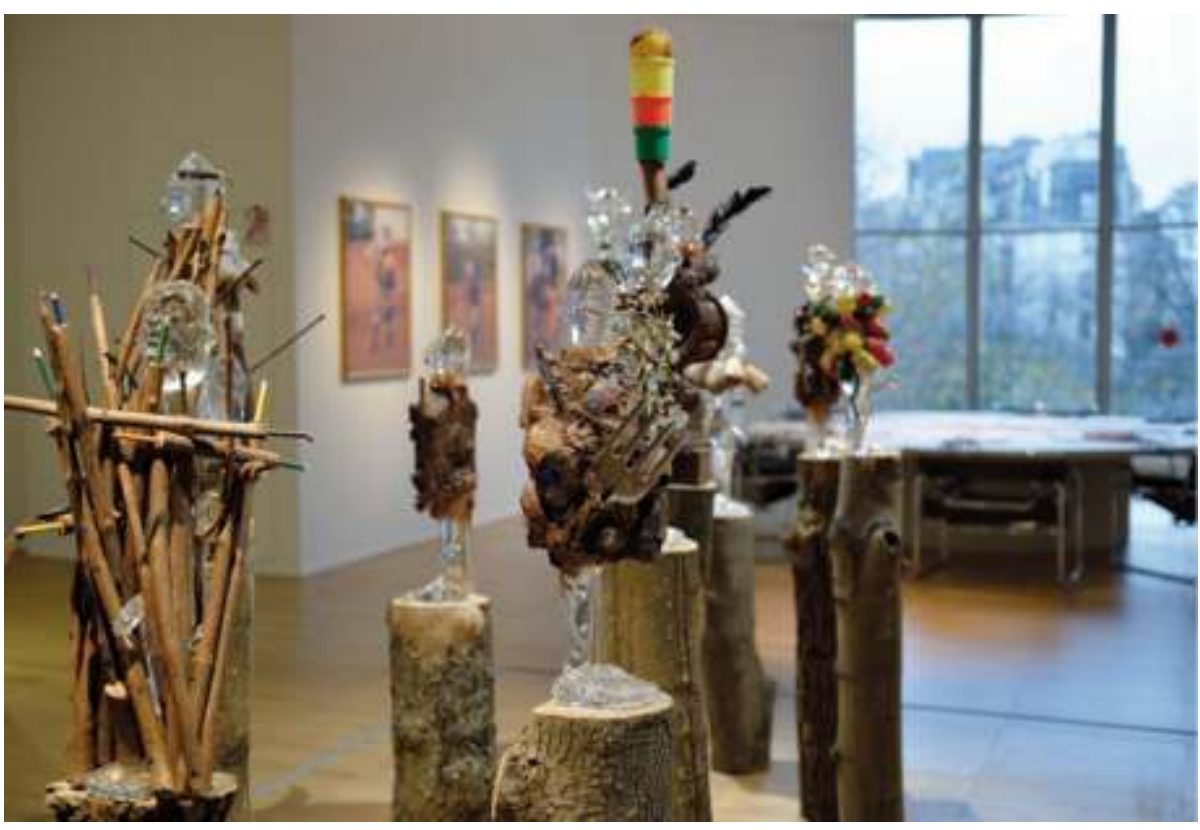

(c) MNHN/J-C Domenech 


\section{RÉSUMÉS}

Chaque année, le musée de l'Homme propose une exposition temporaire thématique. Ces rendezvous réguliers sont l'occasion de positionner le musée comme lieu de débat sur les questions de société relatives à l'Homme, à ses origines et à son devenir. Dans un registre spécifique, lié à la réouverture du musée, la première exposition Chroniques d'une renaissance est conçue pour accompagner le public dans sa découverte du nouveau musée, tel un lever de rideau sur sa partie visible et les coulisses de la rénovation.

INDEX

Mots-clés : musée de l'homme, exposition temporaire

\section{AUTEUR}

\section{ANNE STEPHAN}

Responsable des expositions temporaires au musée de l'Homme 